\title{
On an orthonormal polynomial basis in the space $C[-1,1]$
}

\author{
by \\ KRZYSZTOF WoźnIAKOWski (Warszawa)
}

\begin{abstract}
We show that in the space $C[-1,1]$ there exists an orthogonal algebraic polynomial basis with optimal growth of degrees of the polynomials.
\end{abstract}

1. Introduction. The purpose of this work is to prove the following theorem:

TheOREM 1. For every $\varepsilon>0$ in the space $C[-1,1]$ there exists a basis which consists of orthogonal algebraic polynomials $t_{n}$ such that $\operatorname{deg} t_{n} \leq$ $(1+\varepsilon) n$. The orthogonality is with respect to the Lebesgue measure.

The classical negative result stating that for a polynomial basis $\left(t_{n}\right)$ in the space $C[-1,1]$ we cannot have $\operatorname{deg} t_{n}=n$ is due to Faber [2] and had been obtained before the notion of Banach space was introduced into modern analysis. Then much later A. A. Privalov [9] showed that the system $\left(t_{n}\right)$ cannot satisfy the inequality $\operatorname{deg} t_{n} \leq\left(1+\varepsilon_{n}\right) n$ with $\varepsilon_{n} \rightarrow 0$. In this respect our result is optimal. On the other hand analogous problems for trigonometric polynomials have been solved earlier. The above mentioned paper of Privalov also dealt with the trigonometric case - actually the main ingredient of the proof used properties of trigonometric polynomials. The review of the work done on this problem before 1990 is contained in [13].

The trigonometric case is now completely understood. Some more recent partial positive results in this case were obtained in [10], [11], [16] and [8], and then the complete solutions (with orthogonality property) were given in [7] and [17]. The former work was based on [8] and used the wavelet theory techniques, the latter was based on very close ideas. There were however some problems with transference of these constructions to the algebraic case. It was possible to get bases in the space $C[-1,1]$ satisfying the condition $\operatorname{deg} t_{n} \leq(1+\varepsilon) n$ but orthogonal with respect to some weights (see [5], [4] and [3]). We overcome this difficulty and get orthogonality with respect to Lebesgue measure. After the research presented in this note was completed

2000 Mathematics Subject Classification: 46B15, 46E15, 41A10. 
I learned about the preprint [12] where Theorem 1 is proved by a different method.

The main idea of our work is to start with an appropriate basis of even trigonometric polynomials in $C[-\pi, \pi]$ and perform the orthonormalization procedure with respect to the weight $\sin x$. Thus up to the last moment we will work with trigonometric polynomials.

2. Basis of even polynomials. Let us introduce functions which will serve as an estimate of decay. For $s>0, N=1,2, \ldots$ and $z \in \mathbb{R}$ we define a function $F(s, N, z)(\cdot)$ by the formula

$$
F(s, N, z)(x)= \begin{cases}\sqrt{N} & \text { for }|x-z|<N^{-1} \\ N^{1 / 2-s}|x-z|^{-s} & \text { for }|x-z| \geq N^{-1}\end{cases}
$$

In this section we prove the following result which is the starting point of the construction of an algebraic polynomial basis.

TheOREM 2. For every $\varepsilon>0$ in the space $C[0, \pi]$ there exists a system $\left(u_{n}\right)_{n=0}^{\infty}$ of real trigonometric polynomials of the form $\sum a_{j} \cos j t$ such that:

(i) The system $\left(u_{n}\right)_{n=0}^{\infty}$ is orthonormal with respect to the Lebesgue measure and dense in the space $C[0, \pi]$.

(ii) We have $\operatorname{deg} u_{n} \leq(1+\varepsilon) n$,

(iii) The functions $\left(u_{n}\right)_{n=0}^{\infty}$ can be reordered as $\left(\left(u_{m}^{l}\right)_{m=0}^{a(l)-1}\right)_{l=0}^{\infty}$ for appropriate integers $a(l)$ so that for some $s>3$ we have

$$
\left|u_{m}^{l}\right| \leq C(s) F\left(s, a(l), \mu_{m}^{l}\right)
$$

where $\mu_{m}^{l}=\pi m / a(l)$ for $m=0, \ldots, a(l)-1$.

(iv) There exist orthogonal trigonometric polynomials $\left(f_{m}^{l}\right)_{m=0}^{b(l)}$ where $b(l)=\sum_{k=0}^{l} a(k)$ such that

$$
\begin{aligned}
\operatorname{span}\left(f_{m}^{l}\right)_{m=0}^{b(l)-1} & =\operatorname{span}\left(\left(u_{m}^{k}\right)_{m=0}^{a(k)-1}\right)_{k=0}^{l}, \\
\left\|f_{m}^{l}\right\|_{2} & =1, \\
\left|f_{m}^{l}(x)\right| & \leq C(s) F\left(s, b(l), \nu_{m}^{l}\right)(x),
\end{aligned}
$$

where $\nu_{m}^{l}=\pi m / b(l)$ for $m=0, \ldots, b(l)-1$. Moreover there exists a constant $C$ such that for every $l$ we have $b(l) \leq C a(l)$.

Philosophically we take an appropriate basis of trigonometric polynomials in $C(\mathbb{T})$ and by suitable simple linear combinations we get a basis in the subspace of even functions in $C[-\pi, \pi]$. Many bases of trigonometric polynomials have been constructed (see e.g. [17] and [7]). Also bases of algebraic polynomials are known (see e.g. [3], [4] and earlier [5]). The trouble is that those bases do not have sufficient decay. Only for $\varepsilon>5 / 3$ (actually $>1$ ) we can start with polynomials $\psi_{j, k}(x)$ described in Section 8.4 of [15] and 
note that $u_{j, k}=\psi_{j, k}+\psi_{j, 2^{j}-k-1}$ are even functions. One also easily checks that $u_{j, k}$ with $j=0,1, \ldots$ and $k=0,1, \ldots, 2^{j-1}-1$ form an orthogonal basis in the space of even functions satisfying all requirements of Theorem 2 . Thus we will provide a proof of this theorem. Since its main ideas follow the construction from [16] we will be rather brief.

The proof of the theorem is done in two steps. We start with an elementary proposition which now belongs to the folklore:

Proposition 1. There exists a function $\alpha: \mathbb{R} \rightarrow \mathbb{R}_{+}$such that

(i) $\alpha \in C^{\infty}(\mathbb{R})$,

(ii) $\alpha(x)=0$ when $x \leq 0 ; \alpha(x)=1$ when $x \geq 1$ and $\alpha(x)>0$ when $x \in(0,1)$,

(iii) $\alpha^{2}(1-x)+\alpha^{2}(x)=1$ for $x \in \mathbb{R}$.

Proof. Take any function $\widetilde{\alpha}: \mathbb{R} \rightarrow \mathbb{R}_{+}$such that conditions (i)-(ii) hold. Then

$$
\alpha(x)=\frac{\widetilde{\alpha}(x)}{\sqrt{\widetilde{\alpha}^{2}(1-x)+\widetilde{\alpha}^{2}(x)}}
$$

is our required function.

We define the function $W(x)$ (we drop the index $k$ for convenience) on $\mathbb{R}$ by the formula

$$
W(x)= \begin{cases}\alpha(4 x-1) & \text { for } x \in[1 / 4,1 / 2], \\ \alpha(2-2 x) & \text { for } x \in[1 / 2,1], \\ (-1)^{k} W(-x) & \text { for } x \in[-1,-1 / 4], \\ 0 & \text { otherwise. }\end{cases}
$$

and the function $V(x)$ by the formula

$$
V(x)= \begin{cases}\alpha(2 x-1 / 2) & \text { for } x \in[1 / 4,3 / 4], \\ \alpha(5 / 2-2 x) & \text { for } x \in[3 / 4,5 / 4], \\ (-1)^{k} V(-x) & \text { for } x \in[-5 / 4,-1 / 4], \\ 0 & \text { otherwise. }\end{cases}
$$

To make further formulas more readable for $l \in \mathbb{N}$ let us put

$$
\begin{aligned}
d w(l) & =3 \cdot 2^{l-1}, & d v(l) & =2^{l+1}, \\
D w(l) & =3 \cdot 2^{l}, & D v(l) & =2^{l+2} .
\end{aligned}
$$

Now we define the families of functions $w_{j}^{l}$ and $v_{j}^{l}$ on $\mathbb{T}$ (assuming that the same $k$ is used for any given family) in the following way: for $l>0$ we put

$$
\widehat{w}_{0}^{l}(n)=W\left(n / 2^{l+1}\right),
$$

and then

$$
\begin{aligned}
w_{j}^{l}(t) & =w_{0}^{l}\left(t-x_{j}^{l}\right), \quad j=1, \ldots, d w(l)-1, \quad \text { where } \\
x_{j}^{l} & =2 \pi j / d w(l) .
\end{aligned}
$$


To define families $v_{j}^{l}$ we first put

$$
\widehat{v}_{0}^{l}(n)=V\left(n / 2^{l+1}\right),
$$

and then

$$
\begin{aligned}
v_{j}^{l}(t) & =v_{0}^{l}\left(t-y_{j}^{l}\right), \quad j=1, \ldots, d v(l)-1, \quad \text { where } \\
y_{j}^{l} & =2 \pi j / d v(l) .
\end{aligned}
$$
define

Let us also introduce the Riesz projections. For $f=\sum_{n=-\infty}^{\infty} a_{n} e^{i n t}$ we

$$
\begin{aligned}
& R_{+}(f)(t)=\sum_{n=1}^{\infty} a_{n} e^{i n t}, \\
& R_{-}(f)(t)=\sum_{n=-\infty}^{1} a_{n} e^{i n t} .
\end{aligned}
$$

We have the following proposition, part of which is a particular case of Lemma 1.4 from [16].

Proposition 2. The functions $\left(w_{j}^{l}\right)$ and $\left(v_{j}^{l}\right)$ have the following properties:

(i) If $l>0$ then $w_{0}^{l}$ and $v_{0}^{l}$ are trigonometric polynomials, even and real for $k$ even and odd and purely imaginary if $k$ is odd.

(ii) We have $\left\|w_{j}^{l}\right\|_{2}=d w(l),\left\|v_{j}^{l}\right\|_{2}=d v(l)$ and

$$
\begin{aligned}
\left|R_{-}\left(w_{j}^{l}\right)(t)\right|,\left|R_{+}\left(w_{j}^{l}\right)(t)\right|,\left|w_{j}^{l}(t)\right| & \leq C(s) F\left(s, 2^{l}, x_{j}^{l}\right), \\
\left|R_{-}\left(v_{j}^{l}\right)(t)\right|,\left|R_{+}\left(v_{j}^{l}\right)(t)\right|,\left|v_{j}^{l}(t)\right| & \leq C(s) F\left(s, 2^{l}, y_{j}^{l}\right) .
\end{aligned}
$$

(iii) For every $l$ both families $\left(w_{j}^{l}\right)$ and $\left(v_{j}^{l}\right)$ consist of orthogonal polynomials.

(iv) We have

$$
\begin{gathered}
\operatorname{span}\left(w_{j}^{l}\right)_{j=0}^{d w(l)-1}=W_{l}^{0} \oplus W_{l}^{1}, \\
\operatorname{span}\left(v_{j}^{l}\right)_{j=0}^{d v(l)-1}=V_{l}^{0} \oplus V_{l}^{1},
\end{gathered}
$$

where

$$
\begin{aligned}
W_{l}^{0}= & \operatorname{span}\left\{e^{i n t} \alpha\left(\left(n+2^{l+1}\right) / 2^{l}\right)\right. \\
& \left.+(-1)^{k} e^{i(n+D w(l)) t} \alpha\left(\left(-n-2^{l}\right) / 2^{l}\right)\right\}_{n=-2^{l+1}}^{-2^{l}-1}, \\
W_{l}^{1}= & \operatorname{span}\left\{e^{i n t} \alpha\left(\left(-n+2^{l-1}\right) / 2^{l-1}\right)\right. \\
& \left.+(-1)^{k} e^{i(n+d w(l) t} \alpha\left(\left(2^{l}+n\right) / 2^{l-1}\right)\right\}_{n=-2^{l}}^{-2^{l-1}-1}, \\
V_{l}^{0}= & \operatorname{span}\left\{e^{i n t} \alpha\left(\left(5 \cdot 2^{l-1}+n\right) / 2^{l}\right)\right. \\
& \left.+(-1)^{k} e^{i\left(n+2^{l+2}\right) t} \alpha\left(\left(-3 \cdot 2^{l-1}-n\right) / 2^{l}\right)\right\}_{n=-5 \cdot 2^{l-1}}^{-3 \cdot 2^{l-1}-1},
\end{aligned}
$$




$$
\begin{aligned}
V_{l}^{1}= & \operatorname{span}\left\{e^{i n t} \alpha\left(\left(-n-2^{l-1}\right) / 2^{l}\right)\right. \\
& \left.+(-1)^{k} e^{i\left(n+2^{l+1}\right) t} \alpha\left(\left(3 \cdot 2^{l-1}+n\right) / 2^{l}\right)\right\}_{n=-3 \cdot 2^{l-1}}^{-2^{l-1}-1}
\end{aligned}
$$

Proof. (i) This is obvious and follows directly from the definitions of the functions $W, w_{j}^{l}, V$ and $v_{j}^{l}$.

(ii) The first part of (ii) is elementary and follows from the definitions of $w_{j}^{l}, v_{j}^{l}$ and from Proposition 1(iii) using calculations with Fourier coefficients. For the second part let us start with $w_{0}^{l}$. Using the Poisson Summation Formula we get

$$
w_{0}^{l}(t)=2^{l+1} \sum_{s=-\infty}^{\infty} W^{\vee}\left((t+2 \pi s) 2^{l+1}\right)
$$

where ${ }^{v}$ is the inverse Fourier transform. From the definition of the functions $\alpha$ and $W$ it clearly follows that $W \in C^{\infty}(\mathbb{R})$ so we have $W^{\vee}(t) \leq 1 / t^{s}$ for $t>t_{0}(s)$ and of course $\left|W^{\vee}\right| \leq C$. The same argument holds for $v_{0}^{l}$ and for all Riesz projections. From this and (12) we easily get (ii).

(iii) For the family $\left(w_{j}^{l}\right)$ this was shown in [16] using calculations with Fourier coefficients, and the proof for the family $\left(v_{j}^{l}\right)$ is fully analogous. It also follows directly from the Poisson Summation Formula and the fact that

$$
\left(W^{2}\right)^{\vee}(8 \pi j / 3)=\left(V^{2}\right)^{\vee}(2 \pi j)=0 \quad \text { for } j \in \mathbb{Z} \backslash\{0\} .
$$

(iv) In $[16]$ it has been shown that considering the sums

$$
\sum_{j=0}^{d w(l)-1} e^{i n 2 \pi j / d w(l)} w_{j}^{l}(t)
$$

for $n=-2^{l+1}, \ldots,-2^{l-1}-1$ we can get all the functions in formulas (8) and (9). Similarly using the sums

$$
\sum_{j=0}^{d v(l)-1} e^{i n 2 \pi j / d v(l)} v_{j}^{l}(t)
$$

for $n=-5 \cdot 2^{l-1}, \ldots,-2^{l-1}-1$ we can get all the functions in formulas (10) and (11). Counting dimensions and taking into account that none of the functions from (8)-(11) is equal to zero we get our claim.

Now consider the families of functions

$$
\begin{gathered}
\left\{w_{0}^{l}\right\} \cup\left\{w_{j}^{l}(t)+w_{d w(l)-j}^{l}\right\}_{j=1}^{d w(l) / 2-1} \cup\left\{w_{d w(l) / 2}^{l}\right\}, \\
\left\{v_{0}^{l}\right\} \cup\left\{v_{j}^{l}(t)+v_{d v(l)-j}^{l}\right\}_{j=1}^{d v(l) / 2-1} \cup\left\{v_{d v(l) / 2}^{l}\right\}, \\
\left\{w_{j}^{l}(t)-w_{d w(l)-j}^{l}\right\}_{j=1}^{d w(l) / 2-1}, \\
\left\{v_{j}^{l}(t)-v_{d v(l)-j}^{l}\right\}_{j=1}^{d v(l) / 2-1} .
\end{gathered}
$$

We have the elementary 
Proposition 3. If $k$ is even (resp. odd) the functions in formulas (13) and (14) are even (resp. odd) (i.e. cosine (resp. sine) polynomials) and those in formulas (15) and (16) are odd (resp. even) (i.e. sine (resp. cosine) polynomials). Moreover the functions in (13) and (15) (resp. (14) and (16)) are mutually orthogonal.

Proof. Observe that $w_{j}^{l}(t)=w_{0}^{l}\left(t-x_{j}^{l}\right), w_{d w(l)-j}^{l}(t)=w_{0}^{l}\left(t+x_{j}^{l}\right), v_{j}^{l}(t)=$ $v_{0}^{l}\left(t-y_{j}^{l}\right), v_{d v(l)-j}^{l}(t)=v_{0}^{l}\left(t+y_{j}^{l}\right)$ for all $l>0$ and use Proposition 2(i). For orthogonality use Proposition 2(iii).

Now for a given $\varepsilon>0$ fix a sequence of integers $\left(N_{k}\right)_{k=0}^{\infty}$ such that:

$$
\begin{aligned}
1+\varepsilon / 2 & \leq N_{k+1} / N_{k} \leq 1+\varepsilon, \\
N_{k+1}-N_{k} & =2^{l(k)} \quad \text { for some } l(k) \in \mathbb{N}, \\
N_{k+1}-N_{k} & =2^{\varepsilon(k)}\left(N_{k}-N_{k-1}\right) .
\end{aligned}
$$

where $\varepsilon(k)=0,1$; one can easily see that this is possible. For any positive integer $l$ we introduce the operator

$$
S(l)(f)=e^{-i l t} R_{-}(f)+e^{i l t} R_{+}(f) .
$$

Using this operator and the above sequence of integers we define a new system. For $k \geq 1$, if in (19) we have $\varepsilon(k)=1$ we define

$$
\widetilde{u}_{j}^{k}=S\left(N_{k}-2^{l(k)}\right) w_{j}^{l(k)}
$$

and if in (19) we have $\varepsilon(k)=0$ we define

$$
\widetilde{u}_{j}^{k}=S\left(N_{k}-3 \cdot 2^{l(k)}\right) v_{j}^{l(k)} .
$$

In both cases we have $j=0,1, \ldots, N_{k+1}-N_{k-1}-1$.

In addition we define functions $\left(\widetilde{u}_{j}^{0}\right)_{j=0}^{N_{0}+N_{1}-1}$ by setting

$$
\widetilde{u}_{j}^{0}(t)= \begin{cases}e^{i n t} & \text { if } j=2 n, \\ e^{-i(n+1) t} & \text { if } j=2 n+1,\end{cases}
$$

for $0 \leq j \leq 2 N_{0}-1$, and for $2 N_{0} \leq j \leq 2 N_{0}+2^{l(0)}-1=N_{0}+N_{1}-1$ we set

$$
\widetilde{u}_{j}^{0}(t)=e^{i n t} \alpha\left(\left(n+N_{1}\right) / 2^{l(0)}\right)+e^{i\left(n+N_{0}+N_{1}\right) t} \alpha\left(-\left(n+N_{0}\right) / 2^{l(0)}\right)
$$

where $n=j-3 N_{0}-2^{l(0)}$. We denote the spaces spanned by the above two families by $B_{0}^{1}$ and $B_{0}^{0}$ respectively.

We also introduce the functions

$$
H_{k}(x)= \begin{cases}1 & \text { for }|t| \leq 1, \\ \alpha\left(\left(N_{k+1}-|x| N_{k}\right) 2^{-l(k)}\right) & \text { for }|x| \in\left[1, N_{k+1} / N_{k}\right], \\ 0 & \text { otherwise, }\end{cases}
$$

and the functions $g_{0}^{k}$ defined by the condition

$$
\widehat{g}_{0}^{k}(n)=H_{k}\left(n / N_{k}\right) .
$$


Now if $k$ is even we put

$$
h_{0}^{k}=g_{0}^{k},
$$

and if $k$ is odd we put

$$
h_{0}^{k}(t)=g_{0}^{k}\left(t-\pi /\left(N_{k+1}+N_{k}\right)\right) .
$$

Then in both cases we define $h_{j}^{k}$ as

$$
\begin{aligned}
h_{j}^{k}(t) & =h_{0}^{k}\left(t-z_{j}^{k}\right), \quad j=1, \ldots, N_{k+1}+N_{k}-1, \quad \text { where } \\
z_{j}^{k} & =2 \pi j /\left(N_{k+1}+N_{k}\right) .
\end{aligned}
$$

We define the following families of functions:

- for $k$ even

$$
\begin{gathered}
\left\{h_{0}^{k}\right\} \cup\left\{h_{j}^{k}+h_{\left(N_{k+1}+N_{k}\right)-j}^{k}\right\}_{j=1}^{\left(N_{k+1}+N_{k}\right) / 2-1} \cup\left\{h_{\left(N_{k+1}+N_{k}\right) / 2}^{k}\right\} \\
\left\{h_{j}^{k}-h_{\left(N_{k+1}+N_{k}\right)-j}^{k}\right\}_{j=1}^{\left(N_{k+1}+N_{k}\right) / 2-1}
\end{gathered}
$$

- for $k$ odd

$$
\begin{aligned}
& \left\{h_{j}^{k}+h_{\left(N_{k+1}+N_{k}\right)-j-1}^{k}\right\}_{j=0}^{\left(N_{k+1}+N_{k}\right) / 2-1}, \\
& \left\{h_{j}^{k}-h_{\left(N_{k+1}+N_{k}\right)-j-1}^{k}\right\}_{j=0}^{\left(N_{k+1}+N_{k}\right) / 2-1} .
\end{aligned}
$$

We have the following proposition.

Proposition 4. (i) The operators $S(l)$ map even (resp. odd) functions into even (resp. odd) functions.

(ii) The functions given by (24) and (26) are even and the functions given by (25) and (27) are odd.

(iii) The functions $\widetilde{u}_{j}^{k}$ satisfy the estimate

$$
\left|\widetilde{u}_{j}^{k}\right| \leq C(s) F\left(s, 2^{l}, \xi_{j}^{k}\right)
$$

where $\xi_{j}^{k}$ is equal to $x_{j}^{l}$ or $y_{j}^{l}$, depending on the definition of $\widetilde{u}_{j}^{k}$ (those points are defined in (2) and (3)), and the functions $h_{j}^{k}$ satisfy

$$
\left|h_{j}^{k}(t)\right| \leq C(s) F\left(s, 2^{l}, z_{j}^{k}\right)
$$

where the points $z_{j}^{k}$ are defined in (23).

(iv) The functions $\left(\widetilde{u}_{j}^{k}\right)$ are mutually orthogonal.

(v) The functions $\left(h_{j}^{k}\right)$ are mutually orthogonal.

(vi) We have

(28) $\operatorname{span}\left(h_{j}^{k}\right)_{j=0}^{N_{k}+N_{k+1}-1}=\operatorname{span}\left(\widetilde{u}_{j}^{0}\right)_{j=0}^{N_{0}+N_{1}-1} \oplus \operatorname{span}\left(\left(\widetilde{u}_{j}^{n}\right)_{j=0}^{N_{n+1}-N_{n-1}}\right)_{n=1}^{k}$.

(vii) The system $\left(\widetilde{u}_{j}^{k}\right)$ can be rearranged into a sequence $\left(\widetilde{u}_{n}\right)$ such that $\operatorname{deg} \widetilde{u}_{n} \leq(1 / 2+\varepsilon) n$.

(viii) The system $\left(\widetilde{u}_{n}\right)$ is dense in $C[-\pi, \pi]$. 
Proof. (i) and (ii) are obvious. $S(l)$

(iii) This follows from Proposition 2(ii) and the definition of the operators

(iv) When we apply the operators $S(l(k))$ to formulas (8)-(11) we see that for $k>0$,

$$
\operatorname{span}\left\{\widetilde{u}_{j}^{k}\right\}_{j=0}^{N_{k+1}-N_{k-1}-1}=B_{k}^{0} \oplus B_{k}^{1}
$$

where for $k=0$ the spaces are defined above and for $k \geq 0$ they are given as

$$
\begin{aligned}
B_{k}^{0} & =\operatorname{span}\left\{\gamma(n) e^{i n t}+(-1)^{l} \beta(m) e^{i m t}\right\}, \\
B_{k+1}^{1} & =\operatorname{span}\left\{\beta(m) e^{i n t}+(-1)^{k+1} \gamma(n) e^{i m t}\right\},
\end{aligned}
$$

where $m=n+N_{k}+N_{k+1}$ with $\gamma(n), \beta(m)$ given by the function $\alpha$ and satisfying $\gamma^{2}(n)+\beta^{2}(m)=1$. As it is obvious that $\widetilde{u}_{j}^{k} \perp \widetilde{u}_{i}^{p}$ if $|j-i|>1$ and $B_{k}^{1} \perp B_{k+1}^{0}$ we get orthogonality. Moreover by induction we get

$$
\bigoplus_{m=0}^{k}\left(B_{m}^{0} \oplus B_{m}^{1}\right)=\operatorname{span}\left\{e^{i j t}\right\}_{j=-N_{k}}^{N_{k}-1} \oplus B_{k}^{0} .
$$

(v) The proof is analogous to the above proof of (iv).

(vi) Observe using (29) that $h_{0}^{k} \in \bigoplus_{m=0}^{k}\left(B_{m}^{0} \oplus B_{m}^{1}\right)$ and both spaces in (28) are invariant with respect to rotations of $\mathbb{T}$ by $\Delta=2 \pi /\left(N_{k}+N_{k+1}\right)$.

(vii) This follows immediately from the definition of the operator $S$ and the estimates for the degree of the functions $v_{0}^{l}$ and $w_{0}^{l}$ which are clear from the definitions.

(viii) This follows immediately from (29).

Now for $\left(f_{m}^{l}\right)$ in Theorem 2 we take all functions appearing in formulas $(24)$ and $(26)$ restricted to $[0, \pi]$. For $\left(u_{m}^{l}\right)$ we take the functions $\widetilde{u}_{j}^{k}+\widetilde{u}_{N_{k+1}-N_{k-1}-1-j}^{k}$ also restricted to $[0, \pi]$.

3. Orthogonalization. Let us start by recalling a general lemma about matrices. Various versions of this lemma are known in the literature (see e.g. [6]) but we will present the sharp version due to A. G. Baskakov [1].

Lemma 5. Let $A=A^{*}=\left(a_{i, j}\right)_{i, j=0}^{N}$ be a matrix for which

$$
\|A\|_{2},\left\|A^{-1}\right\|_{2} \leq C
$$

and

$$
\left|a_{i, j}\right| \leq C_{1} /(|i-j|+1)^{s}
$$

for some $s>1$. (The symbol $\|A\|_{p}$ denotes the norm of $A$ as operator on $l_{p}^{N+1}$.) Then for $A^{-1}=\left(c_{i, j}\right)_{i, j=0}^{N}$ we have the estimate

$$
\left|c_{i, j}\right| \leq C\left(C_{0}, C_{1}, s\right) /(|i-j|+1)^{s} .
$$


In this section we will work in the following framework: We have a sequence $\left(\xi_{n}\right)_{n=0}^{N}$ of even trigonometric polynomials of degree $\leq C N$, orthonormal on $[0, \pi]$. We have a sequence of points $z_{0}, z_{1}, \ldots, z_{N}$ in the interval $[0, \pi]$ such that

$$
z_{-1}=0<z_{0}<z_{1}<\ldots<z_{N}<z_{N+1}=\pi
$$

and

$$
\frac{\alpha}{N+1} \leq \min _{k=-1, \ldots, N}\left(z_{k+1}-z_{k}\right) \leq \max _{k=-1, \ldots, N}\left(z_{k+1}-z_{k}\right) \leq \frac{\beta}{N+1}
$$

for some $0<\alpha \leq \beta$ independent of $N$. We assume that

$$
\left|\xi_{n}(x)\right| \leq C F\left(s, N, z_{n}\right)(x)
$$

for some $s>3$ and $n=0,1, \ldots, N$ (see (1)).

Lemma 6. With the above notation we have

$$
\int_{0}^{\pi}\left|\frac{\xi_{i}(x)}{\sqrt{\sin z_{i}}} \cdot \frac{\xi_{j}(x)}{\sqrt{\sin z_{j}}}\right| \sin x d x \leq C(|i-j|+1)^{1 / 2-s} .
$$

Proof. Let $a_{i, j}$ denote the integral on the left hand side of (35). Let us start with a simple property of the function $F\left(s, N, y_{0}\right)$. From the definition of $F$ it easily follows that

$$
\begin{aligned}
\int_{-\infty}^{\infty} F( & \left.s, N, x_{0}\right)(x) F\left(s, N, y_{0}\right)(x) d x \\
& \leq 2 \int_{\left(x_{0}+y_{0}\right) / 2}^{\infty} F\left(s, N, x_{0}\right)(x) F\left(s, N, y_{0}\right)(x) d x \\
& \leq 2 F\left(s, N, x_{0}\right)\left(\frac{x_{0}+y_{0}}{2}\right) \int_{\left(x_{0}+y_{0}\right) / 2}^{\infty} F\left(s, N, y_{0}\right)(x) d x \\
& \leq \frac{C}{\sqrt{N}} F\left(s, N, x_{0}\right)\left(\frac{x_{0}+y_{0}}{2}\right)=\frac{C}{\sqrt{N}} F(s, N, 0)\left(\frac{x_{0}-y_{0}}{2}\right) \\
& \leq \frac{C(s)}{\sqrt{N}} F(s, N, 0)\left(x_{0}-y_{0}\right) .
\end{aligned}
$$

From (34) we clearly have

$$
a_{i, j} \leq \int_{0}^{\pi} \frac{F\left(s, N, z_{i}\right)(x) F\left(s, N, N, z_{j}\right)(x)}{\sqrt{\sin z_{i}} \sqrt{\sin z_{j}}} \sin x d x .
$$

Since for $x, z \in[0, \pi]$ we have $\sin x \leq \sin z+(x-z) \cos z$ we get

$$
\frac{F(s, N, z)(x) \sqrt{\sin x}}{\sqrt{\sin z}} \leq F(s, N, z) \sqrt{1+|x-z| \cdot|\cot z|}
$$


From (33) we infer that $\left|\cot z_{i}\right| \leq C N$ for $i=0,1, \ldots, N$. Since

$$
F\left(s, N, z_{i}\right) \sqrt{1+C N\left|x-z_{i}\right|} \leq C F\left(s-1 / 2, N, z_{i}\right)
$$

from the above observations we infer that

$$
a_{i, j} \leq C^{2} \int_{0}^{\pi} F\left(s-1 / 2, N, z_{i}\right)(x) F\left(s-1 / 2, N, z_{j}\right)(x) d x
$$

so from (36) we get the claim.

Proposition 7. For any sequence $\left(a_{n}\right)_{n=0}^{N}$ of real numbers we have

$$
\int_{0}^{\pi}\left|\sum_{n=0}^{N} a_{n} \frac{\xi_{n}(x)}{\sqrt{\sin z_{n}}}\right|^{2} \sin x d x \sim \sum_{n=0}^{N}\left|a_{n}\right|^{2} .
$$

Proof. Define

$$
A(x)=\sum_{n=0}^{N} a_{n} \frac{\xi_{n}(x)}{\sqrt{\sin z_{n}}} .
$$

We have

$$
\begin{aligned}
I & :=\int_{0}^{\pi} A^{2}(x) \sin x d x \\
& \leq \sum_{i, j}\left|a_{i}\right| \cdot\left|a_{j}\right| \int_{0}^{\pi} \frac{\left|\xi_{i}(x)\right| \cdot\left|\xi_{j}(x)\right|}{\sqrt{\sin z_{i}} \sqrt{\sin z_{j}}} \sin x d x .
\end{aligned}
$$

Using Lemma 6 we get

$$
I \leq C \sum_{i, j}\left|a_{i}\right| \cdot\left|a_{j}\right|(1+|i-j|)^{-s+1 / 2} \leq C(s) \sum_{i}\left|a_{i}\right|^{2}
$$

so we have the upper estimate in (41).

Now we need estimates from below. To get them, to every point $z_{i}$, $i=0, \ldots, N$, we assign an interval

$$
I(i, K)=\left[z_{i}-K / N, z_{i}+K / N\right] \cap[0, \pi],
$$

where $K$ is some constant to be specified later. We claim that

$$
\sum_{i=0}^{N} \sin z_{i} \int_{I(i, K)} A^{2}(x) d x \leq C(K) \int_{0}^{\pi} A^{2}(x) \sin x d x .
$$

Indeed, notice that if

$$
0, \pi \notin I(i, 2 K),
$$


which means that the interval $I(i, K)$ is far from $\{0, \pi\}$, then $\sin z_{i} \leq 2 \sin x$ for $x \in I(i, K)$ so

$$
\sin z_{i} \int_{I(i, K)} A^{2}(x) d x \leq 2 \int_{I(i, K)} A^{2}(x) \sin x d x .
$$

This means that if we denote by $J$ the set of integers $i=0, \ldots, N$ for which (46) holds, then

$$
\sum_{j \in J} \sin z_{j} \int_{I(j, K)} A^{2}(x) d x \leq 4 K \int_{0}^{\pi} A^{2}(x) \sin x d x .
$$

On the other hand if $j \notin J$ then $\operatorname{dist}\left(z_{j}, 0\right) \leq 2 K \beta / N$ or $\operatorname{dist}\left(z_{j}, \pi\right) \leq$ $2 K \beta / N$ so $\sin z_{j} \leq 2 K \beta / N$. Thus

$$
\sin z_{j} \int_{I(j, K)} A^{2}(x) d x \leq 4 \frac{K^{2} \beta}{N^{2}}\left\|A^{2}(\cdot)\right\|_{\infty} .
$$

But from the definition of $\xi_{i}$ we see that $A(\cdot)$ is an even trigonometric polynomial and $\operatorname{deg} A^{2}(\cdot)=2 \operatorname{deg} A(\cdot) \leq C N$. From the well known Bernstein theorem (see e.g. [18]) we infer that

$$
\left(A^{2}(\cdot)\right)^{\prime} \leq C N\left\|A^{2}(\cdot)\right\|_{\infty} .
$$

So if $A^{2}\left(x_{0}\right)=\left\|A^{2}(\cdot)\right\|_{\infty}$ then there exists an interval $I_{0} \ni x_{0}$ with $\left|I_{0}\right| \geq$ $(4 C N)^{-1}$ such that $A^{2}(\cdot) \mid I_{0} \geq\left\|A^{2}(\cdot)\right\|_{\infty} / 2$ and $\sin (\cdot) \mid I_{0} \geq c N^{-1}$. This implies that

$$
\int_{0}^{\pi} A^{2}(x) \sin x d x \geq \int_{I_{0}} A^{2}(x) \sin x d x \geq \frac{c}{N^{2}}\left\|A^{2}(\cdot)\right\|_{\infty} .
$$

Since the number of $j$ 's such that $j \notin J$ is at most $5 K / \alpha$, combining (48) and (49) we get

$$
\sum_{j \notin J} \sin z_{j} \int_{I(j, K)} A^{2}(x) d x \leq C K^{3} \int_{0}^{\pi} A^{2}(x) \sin x d x .
$$

If we put together (47) and (50) we get (45) with a constant $C(K) \leq C K^{3}$. To end the proof it is now enough to estimate from below the expression

$$
\sum_{i=0}^{N} \sin z_{i} \int_{I(i, K)} A^{2}(x) d x .
$$

We clearly have 


$$
\begin{aligned}
& \sum_{i=0}^{N} \sin z_{i} \int_{I(i, K)} A^{2}(x) d x \\
& =\sum_{i=1}^{N} \sin z_{i} \int_{I(i, K)}\left(a_{i} \frac{\xi_{i}(x)}{\sqrt{\sin z_{i}}}+\sum_{\substack{j=0 \\
j \neq i}} a_{j} \frac{\xi_{j}(x)}{\sqrt{\sin z_{j}}}\right)^{2} d x \\
& =\sum_{i=0}^{N} \sin z_{i}\left(\int_{I(i, K)} a_{i}^{2} \frac{\xi_{i}^{2}(x)}{\sin z_{i}} d x\right. \\
& \left.+2 \int_{I(i, K)} \sum_{\substack{j=0 \\
j \neq i}}^{N} a_{i} a_{j} \frac{\xi_{i}(x)}{\sqrt{\sin z_{i}}} \cdot \frac{\xi_{j}(x)}{\sqrt{\sin z_{j}}} d x+\int_{I(i, K)}\left|\sum_{\substack{j=0 \\
j \neq i}}^{N} a_{j} \frac{\xi_{j}(x)}{\sqrt{\sin z_{j}}}\right|^{2} d x\right) \\
& \geq \sum_{i=0}^{N} a_{i}^{2} \int_{I(i, K)} \xi_{i}^{2}(x) d x+2 \sum_{i=0}^{N} \sum_{\substack{j=0 \\
j \neq i}}^{N} a_{i} a_{j} \int_{I(i, K)} \xi_{i}(x) \xi_{j}(x) \frac{\sqrt{\sin z_{i}}}{\sqrt{\sin z_{j}}} d x .
\end{aligned}
$$

Define

$$
d_{i, j}=\int_{I(i, K)} \xi_{i}(x) \xi_{j}(x) \frac{\sqrt{\sin z_{i}}}{\sqrt{\sin z_{j}}} d x
$$

When $z_{j} \notin I(i, K)$, from (38), (39) and (36) we get

$$
\left|d_{i, j}\right| \leq \frac{C(s)}{|i-j|^{s}} *\left(1+|i-j|^{s}\right) \leq \frac{C(s)}{|i-j|^{s-1 / 2}} .
$$

When $z_{j} \in I(i, K)$, i.e. $|i-j| \leq K$, we use the orthogonality of the system $\left(\xi_{i}\right)$ to get

$$
d_{i, j}=\int_{[0, \pi] \backslash I(i, K)} \xi_{i}(x) \xi_{j}(x) \frac{\sqrt{\sin z_{i}}}{\sqrt{\sin z_{j}}} d x .
$$

Estimating $\xi_{j}(\cdot)$ by its maximal value on $[0, \pi] \backslash I(i, K)$ which is less than $C(s) N^{1 / 2} / K^{s}$, and $\sqrt{\sin z_{i}} / \sqrt{\sin z_{j}}$ by $K^{1 / 2}$ and $\int_{0}^{\pi}\left|\xi_{i}(x)\right| d x$ by $C / N^{1 / 2}$ we see that in this case $\left|d_{i, j}\right| \leq 1 / K^{s-1 / 2}$. This gives

$$
\sum_{i=0}^{N} \sin z_{i} \int_{I(i, K)} A^{2}(x) d x \geq \sum_{i=0}^{N} a_{i}^{2} \int_{I(i, K)} \xi_{i}^{2}(x) d x-2 \sum_{i, j=0}^{N}\left|a_{i}\right| \cdot\left|a_{j}\right| \cdot\left|d_{i, j}\right|
$$

and

$$
\left|d_{i, j}\right| \leq \begin{cases}C|i-j|^{s^{\prime}} & \text { for }|i-j|>K \\ C / K^{s^{\prime}} & \text { for }|i-j| \leq K\end{cases}
$$

From (34) and (44) it follows that if $K \rightarrow \infty$ then $\int_{I(i, K)} \xi_{i}^{2}(x) d x \rightarrow C>0$ 
uniformly with respect to $N$. One easily checks that the matrix $D_{K}=\left(\left|d_{i, j}\right|\right)$ maps $\ell_{1}$ into itself and also $c_{0}$ into itself. Moreover both norms tend to zero as $K \rightarrow \infty$. By interpolation we get $\left\|D_{K}\right\|_{2} \rightarrow 0$. We now see that it is possible to take $K$ for which the left-side expression of (53) is always greater than $c \sum_{i=0}^{N}\left|a_{i}\right|^{2}$. This together with (45) ends the proof.

Proposition 8. Let $\left(u_{n}\right)_{n=0}^{\infty}$ be the basis given by Theorem 2 and apply to it the Gram-Schmidt orthogonalization procedure in the space $L_{2}([0, \pi], \sin x d x)$. Then the resulting system $\left(\omega_{n}\right)_{n=0}^{\infty}$ is a Schauder basis in the space $C[0, \pi]$.

Proof. The only thing we really have to prove is that the operators

$$
P_{N} f(x)=\int_{0}^{\pi} K_{N}(x, y) f(y) d y
$$

where

$$
K_{N}(x, y)=\sum_{n=0}^{N} \omega_{n}(x) \omega_{n}(y) \sin y,
$$

considered on the space $C[0, \pi]$ have uniformly bounded norms. We will actually repeat the proof of the fact that the Franklin system is a basis in $C[0,1]$ (see e.g. [14]). To start we will represent the kernel $K_{N}$ in another, more useful form. According to Theorem 2 we have

$$
\operatorname{span}\left(u_{n}\right)_{n=0}^{N}=\operatorname{span}\left(f_{m}^{l}\right)_{m=0}^{b(l)-1} \oplus \operatorname{span}\left(u_{m}^{l+1}\right)_{j=0}^{s},
$$

for some numbers $l$ and $s$. Let the functions on the right side of (54) be called $\left(\xi_{n}\right)_{n=0}^{N}$ and ordered in such a way that

$$
\xi_{n}(x) \leq C(s) F\left(s, N, z_{n}\right)(x)
$$

where $z_{n}$ 's satisfy (33). This can be done because $a(l) \sim b(l) \sim N$, so we really are in the framework described at the beginning of Section 3. From the very definition of $\left(\omega_{n}(x)\right)$ it follows that

$$
K_{N}(x, y)=\sum_{i, j=0}^{N} c_{i, j} \frac{\xi_{i}(x)}{\sqrt{\sin z_{i}}} \frac{\xi_{j}(y)}{\sqrt{\sin z_{j}}} \sin y
$$

for some numbers $c_{i, j}$.

We will estimate the kernel $K_{N}(x, y)$. First observe that Proposition 7 is equivalent to the statement that the self-adjoint matrix $A=\left(a_{i, j}\right)_{i, j=0}^{N}$ given by

$$
a_{i, j}=\int_{0}^{\pi} \frac{\xi_{i}(x)}{\sqrt{\sin z_{i}}} \frac{\xi_{j}(x)}{\sqrt{\sin z_{j}}} \sin x d x
$$

fulfills the estimates

$$
\|A\|_{2},\left\|A^{-1}\right\|_{2} \leq C
$$


Since $P_{N}\left(\xi_{i}\right)=\xi_{i}$ for $i=0,1, \ldots, N$ we infer that $c_{i, j}$ 's in (56) are equal to the entries of the inverse matrix $A^{-1}$. Now we are ready to estimate $\sup _{x} \int_{0}^{\pi}\left|K_{N}(x, y)\right| d y$. Let us start with

$$
\begin{aligned}
\int_{0}^{\pi}\left|\xi_{j}(y)\right| \sin y d y & \leq \int_{0}^{\pi} \mid \xi_{j}(y)\left[\sin z_{j}+\cos z_{j}\left(y-z_{j}\right)\right] d y \\
& \leq \frac{\sin z_{j}}{\sqrt{N}}+C \int_{0}^{\pi} F\left(s, N, z_{j}\right)(y)\left|y-z_{j}\right| d y \\
& \leq \frac{\sin z_{j}}{\sqrt{N}}+\frac{C}{N^{3 / 2}} \leq C \frac{\sin z_{j}}{\sqrt{N}}
\end{aligned}
$$

Moreover we have

$$
\frac{\sin z_{i}}{\sin z_{j}} \leq C N\left|z_{i}-z_{j}\right|
$$

Thus we have

$$
\begin{aligned}
& \int_{0}^{\pi}\left|K_{N}(x, y)\right| d y \\
& \quad \leq \sum_{i, j=0}^{N} \frac{C}{(1+|i-j|)^{s-1 / 2}} \cdot \frac{\left|\xi_{i}(x)\right|}{\sqrt{\sin z_{i}} \sqrt{\sin z_{j}}} \int_{0}^{\pi}\left|\xi_{j}(y)\right| \sin y d y \\
& \quad \leq \sum_{i, j=0}^{N} \frac{C}{(1+|i-j|)^{s-1 / 2}} \cdot \frac{\left|\xi_{i}(x)\right|}{\sqrt{\sin z_{i}} \sqrt{\sin z_{j}}} \frac{\sin z_{j}}{\sqrt{N}} \\
& \quad \leq \sum_{i, j=0}^{N} \frac{C}{(1+|i-j|)^{s-1 / 2}} \cdot \frac{\left|\xi_{i}(x)\right|}{\sqrt{N}} \sqrt{\frac{\sin z_{j}}{\sin z_{i}}}
\end{aligned}
$$

Using (58) and (33) we estimate this sum as

$$
\sum_{i, j=0}^{N} \frac{C \sqrt{|i-j|}}{\sqrt{N}(1+|i-j|)^{s-1 / 2}} F\left(s, N, z_{i}\right)(x) \leq \text { const. }
$$

If we insert (60) into (59) and use the well known formula for the norm of an integral operator on $C[-1,1]$ we get the required estimate.

Completion of the proof of Theorem 1. Let $I$ be the well known operator defined for functions $f:[0, \pi] \rightarrow \mathbb{R}$ and $s \in[-1,1]$ by

$$
\text { If }(s)=f(\arccos s) \text {. }
$$

It is well known and easy that $I$ is an isometry between $C[0, \pi]$ and $C[-1,1]$ and between $L_{2}([0, \pi], \sin x)$ and $L_{2}([-1,1])$. Also $I(\cos n t)$ is a Chebyshev 
polynomial, in particular it is an algebraic polynomial of degree $n$. So from Theorem 2 and Proposition 8 it follows that the system of functions

$$
t_{n}=\omega_{n}(\arccos s)
$$

is as described in Theorem 1.

\section{References}

[1] A. G. Baskakov, Abstract harmonic analysis and asymptotic estimates for elements of inverse matrices, Mat. Zametki 52 (1992), no. 2, 17-26 (in Russian).

[2] G. Faber, Über die interpolatische Darstellung stetiger Funktionen, Jahresber. Deutsch. Math.-Verein. 23 (1914), 192-210.

[3] R. Girgensohn, Polynomial Schauder bases for $C[-1,1]$ with Chebycheff orthogonality, preprint of the GSF-National Research Center for Environment and Health, Institute of Biomathematics and Biometry.

[4] R. Girgensohn and J. Prestin, Lebesgue constants for an orthogonal polynomial Schauder basis, J. Comput. Anal. Appl. 2 (2000), 159-172.

[5] T. Kilgore, J. Prestin and K. Selig, Orthogonal algebraic polynomial Schauder bases of optimal degree, J. Fourier Anal. Appl. 2 (1996), 597-610.

[6] P. G. Lemarié, Base d'ondelettes sur les groupes de Lie stratifiés, Bull. Soc. Math. France 117 (1989), 211-232.

[7] R. A. Lorentz and A. Sahakian, Orthonormal trigonometric Schauder bases of optimal degree for $C(K)$, J. Fourier Anal. Appl. 1 (1994), 103-112.

[8] D. Offin and K. Oskolkov, A note on orthonormal polynomial bases and wavelets, Constr. Approx. 9 (1993), 319-326.

[9] A. A. Privalov, On the growth of degrees of polynomial bases and approximation of trigonometric projections, Mat. Zametki 42 (1987), no. 2, 207-214 (in Russian).

[10] -, On the growth of degrees of polynomial bases, ibid. 48 (1990), no. 4, 69-78 (in Russian).

[11] —, On an orthogonal trigonometric basis, Mat. Sb. 182 (1991), 384-394 (in Russian).

[12] M. Skopina, Orthogonal polynomial Schauder bases for $C[-1,1]$ of optimal degree, preprint.

[13] P. L. Ul'yanov, On some results and problems from the theory of bases, Zap. Nauchn. Sem. Leningrad. Otdel. Mat. Inst. Steklov. (LOMI) 170 (1989), 274-284 (in Russian).

[14] P. Wojtaszczyk, Banach Spaces for Analysts, Cambridge Univ. Press, Cambridge, 1991.

[15] -, A Mathematical Introduction to Wavelets, London Math. Soc. Student Texts 37, Cambridge Univ. Press, Cambridge, 1997.

[16] P. Wojtaszczyk and K. Woźniakowski, Orthonormal polynomial bases in function spaces, Israel J. Math. 75 (1991), 167-191.

[17] K. Woźniakowski, Constructions of unconditional bases in $H_{p}$ spaces, Ph.D. thesis, Polish Academy of Sciences, 1995 (in Polish). 
[18] A. Zygmund, Trigonometric Series, Cambridge Univ. Press, Cambridge, 1959.

Towarzystwo Ubezpieczeń i Asekuracji "Warta" SA

Biuro Aktuarialne

Wydział Matematyki Ubezpieczeniowej

E-mail: kispam@kki.net.pl

Received June 20, 2000

Revised version November 7, 2000 